\title{
Measurement of sub-shot-noise spatial correlations without subtraction of background
}

\author{
Giorgio Brida ${ }^{1}$, Lucia Caspani ${ }^{2}$, Alessandra Gatti ${ }^{2}$, Marco Genovese $^{1}$, Alice Meda ${ }^{1}$, Ivano Ruo Berchera ${ }^{1}$ \\ ${ }^{1}$ INRIM, strada delle Cacce 91, 10135 Torino, Italy. \\ 2 INFM-CNR-CNISM, Universitá dell'Insubria, Via Valleggio 11, 22100 Como, Italy.
}

\begin{abstract}
In this paper we present the measurement of sub-shot-noise spatial correlations without any subtraction of background, a result paving the way to realize sub-shot-noise imaging of weak objects.
\end{abstract}

PACS numbers: 42.50.Ar, 42.50.Dv, 42.50.Lc,03.65.Wj

Quantum properties of the optical field represent a resource of the utmost relevance for the development of quantum technologies, allowing unprecedented results in disciplines ranging from quantum information and metrology [1] to quantum imaging (for recent reviews see 2]). For applications in this latter field, a fundamental tool to be realized is the spatial correlation, at the level of quantum fluctuations, of two optical beams. An example is the high-sensitivity detection of weak objects proposed in 3, which exploits the intrinsic multi-mode quantum correlations of parametric down conversion (PDC) emission, a result that may have disruptive practical applications in biomedical imaging or whenever there is the need of illuminating an object with a low flux of photons 20 . This technique is based on the spatial correlation in the quantum noise of two conjugated branches of PDC emission [4, 5, 6]. The image of a weak absorbing object in one branch, eventually previously hidden in the noise, can be restored by subtracting the strongly correlated spatial noise pattern measured in the other branch. In order to achieve a sensitivity superior than that available with classical techniques, one should be able to reach a subshot-noise (SSN) regime in spatial correlations even in the presence of the unavoidable background noise (electronic noise, scattered light) 3. Nevertheless, up to now, such a result was not yet achieved. Indeed, previous demonstrations of the quantum nature [8, 9] of the spatial correlation of PDC beams were realized only for low photon numbers. In that regime the background noise was dominant, so that a proof-of-principle demonstration of SSN correlation was possible only after correcting the results for the background noise (i.e. by subtracting the variance of the spatial pattern of the background, measured in the absence of PDC). Clearly, such a regime cannot be used for concrete imaging schemes, as the image distribution would remain hidden in the background noise. Similarly, a single-mode sub-shot-noise intensity correlation [10, 11, 12, 13, 14, 15] cannot be used to retrieve high-sensitivity information on the spatial distribution of an object, since the quantum character of the correlation vanishes when one detects small portions of the beams instead of the whole beams. Thus, high sensitivity imaging requires spatial quantum correlations (i.e. SSN intensity correlations between several portions of two twin beams) in a regime where the photon flux

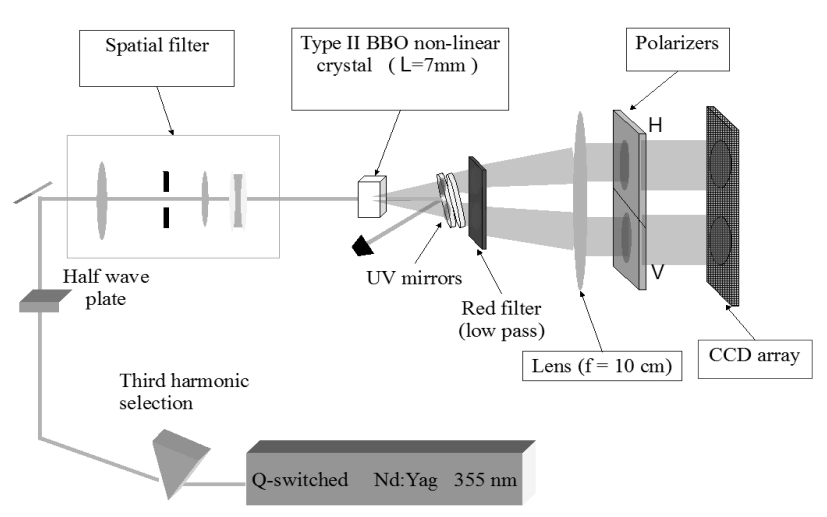

FIG. 1: Experimental setup. A triplicated Nd-Yag laser beam, after spatial filtering, produces type II PDC in a BBO crystal, which is then measured (after eliminating UV beam) by an high sensitivity CCD camera.

is high enough to make the background noise negligible. Purpose of the present letter is to present this achievement: a clear observation of SSN spatial correlation, without any correction for the background noise, is presented and discussed, opening the concrete possibility of realizing imaging with a sensitivity beyond the standard quantum limit.

The strategy we shall follow to reach our goal is that indicated by [3]: the major limitation of the experiment [8] was the presence in each beam of a large excess noise with respect to coherent light, which made SSN correlation fragile with respect to unavoidable experimental imperfections (unbalances, errors in the determination of the symmetry center of the signal-idler distributions) and rapidly deteriorated the correlation for increasing gain. Such an excess noise can be lowered by working in a different regime, i.e. a pump pulse of duration much longer than the typical coherence time of PDC beams, which is on the order of ps for a few mm crystal. As predicted in [3], a nanosecond pump pulse should enable the observation of SSN correlations at a much higher photon number. In our setup (Fig 1) a type II BBO non-linear crystal $(l=7 \mathrm{~mm})$ is pumped by the third harmonic (355 $\mathrm{nm}$ ) of a Q-switched Nd:Yag laser (with pulses of $5 \mathrm{~ns}$, 
$10 \mathrm{~Hz}$ repetition rate and a maximum energy of about $200 \mathrm{~mJ}$ ) after spatial filtering (a lens with a focal length of $50 \mathrm{~cm}$ and a diamond pin-hole, $250 \mu \mathrm{m}$ of diameter), for eliminating the non-gaussian components, and collimation (by a system of lenses to a diameter of $w_{p}=1.25$ $\mathrm{mm}$ ). After eliminating the pump (by a couple of UV mirrors, transparent to the visible, $\mathrm{T}=98 \%$ at $710 \mathrm{~nm}$, and by a low frequency-pass filter, $\mathrm{T}=95 \%$ at $710 \mathrm{~nm}$ ) the far-field of the down converted beams (signal and idler) is imaged onto a CCD camera, by means of a lens $(f=10 \mathrm{~cm})$ in a $f-f$ optical configuration, ensuring that we image the Fourier transform of the crystal exit face. Therefore each transverse mode with wavevector $\mathbf{q}$ of the emission is associated to a point $\mathbf{x}=(\lambda f / 2 \pi) \mathbf{q}$ of the image. We use a $1340 X 400 \mathrm{CCD}$ array, Princeton Pixis400BR (pixel size of $20 \mu \mathrm{m}$ ), with high quantum efficiency $(80 \%)$ and low noise in the read out process. The CCD exposure time is set to $90 \mathrm{~ms}$, thus each image catched in this time window corresponds to the PDC emission generated by a single shot of the laser. After having identified the degenerate emissions (two separated rings in our phase matching configuration), at wavelength $\lambda_{s}=\lambda_{i}=710 \mathrm{~nm}$, by inserting an interference filter, we focus the analysis on two correlated areas of side 2,72 $\mathrm{mm}$ within a $10 \mathrm{~nm}$ bandwidth around it. In each of the two signal-idler regions, the unwanted contribution of frequency components of the orthogonal polarizations other than the degenerate one are eliminated by a polarizer $\mathrm{T}=97 \%$ ). Notice that differently with the setup of [8] the signal and idler beams follow basically the same optical path, so that unbalances are reduced.

In the ideal case of a plane wave pump $\left(\mathbf{q}_{\text {pump }}=0\right)$, the momentum conservation in the microscopic PDC process requires that each pair of signal and idler photons is emitted in symmetric directions $\mathbf{q}_{s}=-\mathbf{q}_{i}$. As a consequence, perfect intensity spatial correlation between any symmetric portions $\mathbf{x}_{s}=-\mathbf{x}_{i}$ of the far-field zone of the signal and idler beams were predicted [4, 5, 6, For a finite pump of waist $w_{p}$, having a distribution of transverse momenta, there is an uncertainty in the relative directions of propagation of twin photons represented by the angular bandwidth of the pump, so that the photon numbers collected from symmetrical portions of the farfield zone are perfectly quantum correlated only when the detection areas are broader than a coherence area (roughly the transverse size of the mode in the far field), whose size $\Delta x_{c o h}^{2}$ depends on the pump angular bandwidth $\lambda / w_{p}, 5$, 6], and on the parametric gain [3, 9, 16]. Thus, we expect a quantum correlation in the number of photons $\widehat{N}_{i}$ and $\widehat{N}_{s}$ detected at any couple of symmetric positions with respect to the center of symmetry, providing our detection areas are larger than the typical coherence area.

The quantity that characterizes the amount of correlation is the noise reduction factor $\sigma$, defined as the variance of the photon number difference $\widehat{N}_{i}-\widehat{N}_{s}$ normalized to the shot noise. In the ideal case of detection areas much larger than the coherence area we have the usual "twin beams" result: 4, 17.

$$
\sigma \equiv \frac{\left\langle\delta^{2}\left(\widehat{N}_{i}-\widehat{N}_{s}\right)\right\rangle}{\left\langle\widehat{N}_{i}+\widehat{N}_{s}\right\rangle}=1-\eta,
$$

where $\eta$ represents the total transmittance of the optical channel, including the quantum efficiency of the detectors. For classical light $\sigma$ is above 1 and reaches the lowest limit in the case of coherent light $\left(\sigma_{c o h}=1\right)$ which is referred to as Shot Noise Level (SNL). Whereas for twin beams $\sigma$ is always smaller than unity (see Eq11). The other relevant quantity is the Fano factor $F_{s(i)} \equiv$ $\left\langle\delta^{2} \widehat{N}_{s(i)}\right\rangle /\left\langle\widehat{N}_{s(i)}\right\rangle$, which represents the fluctuation of the single beam normalized to the shot noise. For PDC, since each beam taken alone has a thermal-like statistics, the Fano factor has the multi-thermal form: $F_{s(i)}=1+E_{n}$, where $E_{n}=\left\langle\widehat{N}_{s(i)}\right\rangle / M$ is called "excess noise" and $M$ is the degeneracy factor, proportional to the number of spatial and temporal modes collected in the measurement.

We estimate the expectation values as averages over a large spatial ensemble (large regions of a single image). Specifically, let us define $N(\mathbf{x})$ the number of photons, registered by the CCD pixel in the position $\mathbf{x}$ of a generic region $R$ of the CCD. $\delta N(\mathbf{x})=N(\mathbf{x})-\langle N(\mathbf{x})\rangle$ is the fluctuation around the mean value, estimated as $\langle N(\mathbf{x})\rangle=(1 / n) \sum_{\mathbf{x}} N(\mathbf{x})$, with $n$ the number of pixels in $R$. We estimate the $\sigma$ parameter by fixing a region $R_{s}$ in the signal portion (136X136 pixels), and searching the corresponding region $R_{i}$ of the idler field that minimizes the experimental noise reduction factor:

$$
\sigma_{e x p}(\xi)=\frac{\left\langle\delta^{2}\left[N_{s}(\mathbf{x})-N_{i}(-\mathbf{x}+\xi)\right]\right\rangle}{\left\langle N_{s}(\mathbf{x})+N_{i}(-\mathbf{x}+\xi)\right\rangle}
$$

where $\xi$ is the displacement vector of $R_{i}$ in the pixel space with respect to the optimal position. In other words, the numerator represents the residual spatial noise of the image in $R_{s}$ after having subtracted pixel-by-pixel the correlated noise pattern in $R_{i} . \sigma_{e x p}(\xi)$ is plotted in Fig.2. Far from the minimum, we are in the situation in which the two regions are completely uncorrelated, and $\sigma_{\text {exp }}$ approaches the Fano factor of the single region (we suppose for simplicity $F_{s} \approx F_{i}$ ). Furthermore, Fig 2 (a) shows that the size of the coherence area is larger than the pixel and the Full Width Half Maximum of the deep gives us $\Delta x_{c o h}$ in terms of pixels. For this image, we estimate $\Delta x_{c o h} \approx 8$ pixels $(160 \mu m)$. Since the SSN can be achieved only for detection areas containing one or more coherence areas, we performed a binning of the pixels, i.e. we group the physical pixels in blocks of dimension $8 X 8$, called superpixels. The number of photons collected in a superpixel is the sum of the photons collected by each original pixel. Our CCD allows to perform the binning 


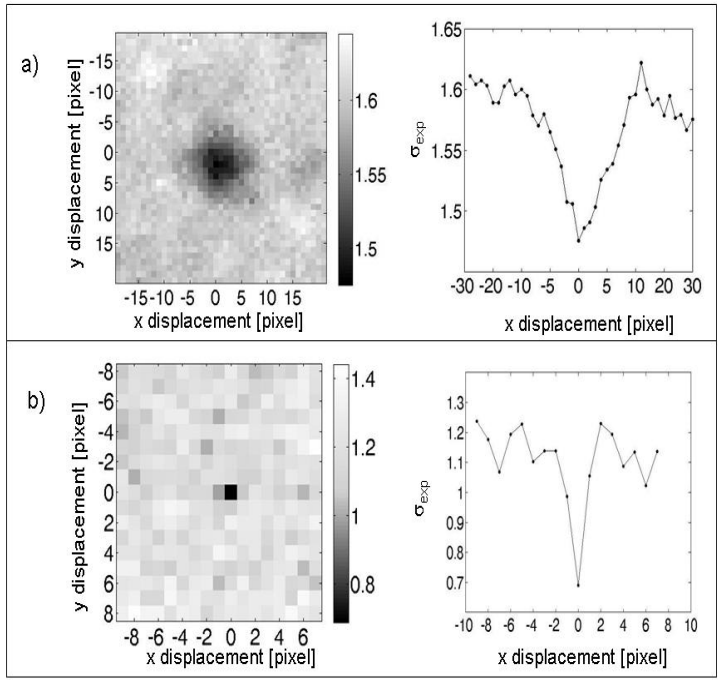

FIG. 2: Example of noise reduction factor $\sigma_{\text {exp }}$, evaluated for a specific image, as a function of the $2 \mathrm{D}$ position of the region $R_{i}$ (left panels). The right panels are sections of the minimum. (a) $\sigma$ is measured using the CCD pixels (side 20 $\mu \mathrm{m})$. Its minimum value stays just 0.15 below the Fano factor ( $\sigma$ evaluated far from the minimum) and is above 1. (b) $\sigma$ is measured using larger pixels (side $160 \mu \mathrm{m}$ ). In this case, the correlation is higher (the gap between the Fano factor and the minimum is about 0.6) and, especially, the minimum is below 1 , i.e. the correlation is sub-shot-noise

operation at the hardware level, also lowering strongly the signal to electronic noise ratio. Fig 2 (b) shows $\sigma_{\text {exp }}$ in the case of binning. The deep $\sigma_{\exp }(0)$, representing the estimate of $\sigma$, is noticeably under the SNL while the FWHM of the coherence area is clearly on the order of the superpixel dimension. We note that, although the operation of binning reduces the spatial statistical ensemble, nevertheless the chosen region $R_{s}$ is large enough to contain about 300 superpixels. This number provides an estimate of the spatial resolution, because it gives the number of details of an image that one would be able to distinguish in an imaging experiment. It is clear 3 that the resolution in this high-sensitivity scheme is limited by the size of the coherence area of PDC, which in principle can be reduced by acting on the pump transverse size (a systematic experimental study of this dependence having been presented in [16]).

Fig 3 presents the minimum values of $\sigma_{\exp }$ plotted as a function of the photon number, where for each data point the center of symmetry of the two images (the position of the minimum of $\sigma_{\text {exp }}$ ) is determined with subpixel resolution, using the algorithm described in [18]. The corresponding Fano factor $F=\left(F_{s}+F_{i}\right) / 2$ is also reported. Each point of the main graph represents the result evaluated for a single image, generated by single laser shot, while in the small insert the data are averaged over several images. The comparison between $F$ and $\sigma$

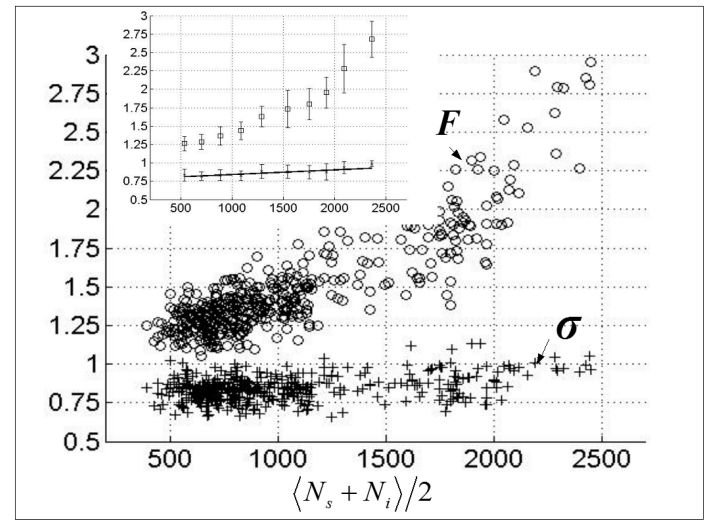

FIG. 3: The noise reduction factor $\sigma_{\text {exp }}$, without any background correction, is plotted versus the mean photon number per pixel. Each point (crosses) in the main window corresponds to the analysis of a single image. The corresponding Fano factor, $F$, is reported by the circles. Several images reach a noise reduction under 0.7 . The insert on the top-left angle presents the same data averaged over tens of images. The equation for the fit is $y=(5.9 \pm 1.5) 10^{-5} x+(0.78 \pm 0.02)$.

gives a measure of the reduction of the noise level that can be observed in a differential measurements, where the intensities pattern of correlated regions are properly subtracted. Although no a posteriori correction for experimental noise and imperfections has been applied, all the fitting line is clearly SSN in the range of photon number considered, with a minimum of 0.82 for a photon number per pixel of 600 (the smallest value for a single shot being 0.66). Also the dispersion of the data is reduced, being almost all the point under the SNL. This figures are further reduced to $\sigma=0.7$ when a $12 \times 12$ binning is used (the smallest value for a single shot being 0.5 ). This two facts represent a fundamental new feature with respect to 8 , where a lot of images were above SNL and a heavy noise correction had been applied on the experimental values of $\sigma$. Our data demonstrate that in our setup the spatial fluctuations of two selected regions, comprehensive of the unavoidable electronic noise and diffuse light, are really correlated at the quantum level, not only in principle, and therefore it is possible to exploit this correlation for real and new application to imaging. For example for an object transmittance $\alpha<15 \%$, following the theoretical prediction of 3 we can estimate that our setup would provide a SNR more than $10 \%$ better than classical light with $8 \times 8$ binning ( $20 \%$ with a $12 \times 12$ binning).

Let us consider the sources of noise that can spoil the spatial correlation. First of all the electronic noise in the read-out and digitalization process is $\Delta=4$ photoelectron/pixel and $\Delta=9$ photoelectron/superpixel in the case of binning. Then, the quantum efficiency of the pixels fluctuates spatially with a standard deviation $\delta \eta=3 \%$. Anyway, this is a very minor effect in our pho- 


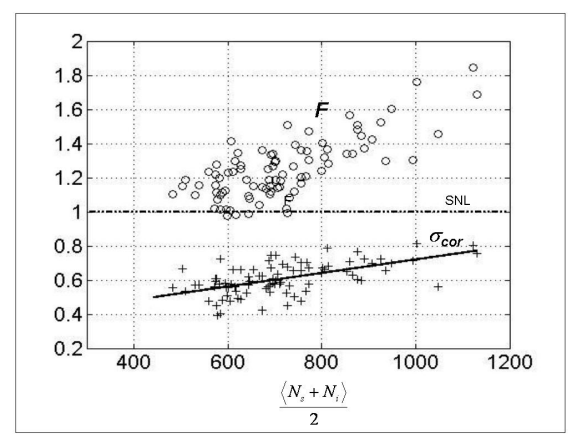

FIG. 4: Noise reduction factor $\sigma_{\text {cor }}$ and Fano factor $F$, corrected for the background noise. Here we present a subset of the same data showed in Fig. 4 once the electronic and the diffuse light noise has been subtracted. The equation for the fit is $y=(3.7 \pm 1.1) 10^{-4} x+(0.38 \pm 0.08)$

ton number regime. On the other side, the fluorescence of the crystal, mirrors and filters, absorbing the high energy pump pulse, together with the residual light of the pump itself, contribute to a background of diffuse light collected by the CCD in each shot. This represents the main source of noise in our present configuration. For the sake of comparison with previous experiments, we can correct for this background noise, acquiring an image where the PDC emission has been suppressed, simply rotating the pump polarization by 90 degrees. Let us define $S_{s(i)}^{2}$ the variance of the spatial fluctuations of this image in the regions $R_{s(i)}$, and $M_{s(i)}$ the mean photon numbers. We have typical values of $S_{s(i)}^{2} \sim 200$ and $M_{s(i)} \sim 120$. The formula for the corrected noise reduction factor is

$$
\sigma_{c o r}(0)=\frac{\left\langle\delta^{2}\left[N_{s}(\mathbf{x})-N_{i}(-\mathbf{x})\right]\right\rangle-S_{s}^{2}-S_{i}^{2}}{\left\langle N_{s}(\mathbf{x})+N_{i}(-\mathbf{x})\right\rangle-M_{s}-M_{i}}
$$

The same correction can be applied to the Fano factors. Fig 4 reports the results applied to a subset (for graphical reasons) of the data of Fig. 3 Some image reaches values of noise reduction of 0.4 and the average is under 0.6 in the lower photon number condition. The Fano factors are reduced as well. These data can be compared with the theoretical threshold for the noise reduction given by Eq.(1). Since we estimate in our setup a total transmission-detection efficiency of $67 \%$, it leads to $\sigma=0.33$. This means that we still have a net margin of improvement of 0.27 . We attribute the gap between the theoretical prediction and the experimental achievement to i) the fact that in our setup the superpixel is just on the order of the coherence area, while the optimal condition for the observation of SSN would require a detection area sensibly larger than the coherence area, and ii) a residual error in the determination of the symmetry center of the signal-idler distributions, because the bigger is the superpixel, the larger is the uncertainty in the evaluation of the symmetry center, even when this is retrieved by using a sub-pixel algorithm, as described in [18.

In conclusion, in this paper we presented our results showing that we reached the condition of sub-shot-noise in spatial correlation without any background subtraction, a fundamental achievement for realizing high sensitivity quantum imaging protocols. Indeed, we obtained a substantial reduction of the noise under SNL in the spatial distribution of a large portion of PDC emission by subtracting the correlated one.

We acknowledge precious advices and discussions with E. Brambilla, L.Lugiato, O. Jedrkiewicz. This work was supported by Compagnia di San Paolo, by PRIN 2007FYETBY, by Regione Piemonte (E14) and by the EC FET under the GA HIDEAS FP7-ICT-221906. We would like to acknowledge that whilst this letter was under referring process a paper presenting similar results, albeit in completely different intensity and detection regime and a configuration not devoted to imaging applications, appeared in this journal [19].

[1] D. Bouwmeester, A. K. Ekert, A. Zeilinger, The Physics of Quantum Information: Quantum Cryptography, Quantum Teleportation, Quantum Computation, (Springer, New York, 2000); M. Genovese, Phys. Rep. 413/6, 319-398 (2005), and references therein.

[2] see the papers in Quantum Imaging, M.I. Kolobov ed.(Springer Verlag, Singapore); A. Gatti et al., in Progress in Optics, edited by E. Wolf (Elsevier NorthHolland, Amsterdam, 2008), Vol. 51, p. 251.

[3] E. Brambilla et al., Phys. Rev. A 77, 053807 (2008).

[4] A. Gatti et al., Phys. Rev. Lett 83, 1763 (1999).

[5] E. Brambilla et al., Eur. Phys. J. D 15, 1277 (2001).

[6] E. Brambilla et al. Phys. Rev. A 69, 023802 (2004).

[7] Y.Fu et al., Opt. Exp 14 (2006) 3942 and ref.s therein.

[8] O. Jedrkievicz et al., Phys. Rev. Lett. 93, 243601 (2004).

[9] O. Jedrkievicz et al., J. Mod. Opt. 53, 575 (2006).

[10] C. D. Nabors and R. M. Shelby, Phys. Rev. A 42, 556 (1990).

[11] P. R. Tapster et al, Phys. Rev. A 44, 3266 (1991).

[12] P. H. Souto Ribeiro et al, Opt. Lett. 22, 1893 (1997).

[13] J. Gao et al, Opt. Lett. 23, 870 (1998).

[14] M.Bondani et al., European Physical Journal - special topics 160, 33 (2008).

[15] T. Iskhakov et al., JETP Lett. 88, 660 (2008).

[16] G.Brida, M. Genovese, A. Meda, E. Predazzi and I. Ruo Berchera, Int. Journ. Quant. Inf. 7 (2009) 139; Journal of Modern Optics, 56 (2009) 201.

[17] G. Brida et al., J. Opt. Soc. Am. B 23, 2185 (2006).

[18] L. Caspani et al., 55, 2025-2043 (2008).

[19] J. Blanchet el al. Phys Rev. Lett. 101, 233604 (2008).

[20] For example, low illumination levels are needed in presence of pigments or other photo-sensible molecules, see e.g. 7]. The sub-shot noise imaging requires a photon 
flux roughly $\sigma$ times smaller than an equivalent classical one, in order to achieve the same signal-to-noise ratio [3]. 